\title{
Coherent and incoherent pumping of electrons in double quantum dots
}

\author{
B. L. Hazelzet, M. R. Wegewijs, T. H. Stoof, and Yu. V. Nazarov \\ Department of Applied Physics, Faculty of Applied Science, Delft University of Technology, Lorentzweg 1, \\ 2628 CJ Delft, The Netherlands
}

(Received 30 December 1999; revised manuscript received 13 November 2000; published 4 April 2001)

\begin{abstract}
We propose an alternative mode of operation of an electron pump consisting of two weakly coupled quantum dots connected to reservoirs. An electron can be transferred within the device at zero bias voltage when it is subjected to electromagnetic radiation, thereby exciting the double dot. The excited state can decay by transferring charge from one lead and to the other lead in one direction. Depending on the energies of the intermediate states in the pumping cycle, which are controlled by the gate voltages, this transport is either incoherent via well-known sequential tunneling processes, or coherent via an inelastic co-tunneling process. The latter mode of operation is possible only when interdot Coulomb charging is important. The dc transport through the system can be controlled by the frequency of the applied radiation. We concentrate on the resonant case, when the frequency matches the energy difference for exciting an electron from one dot into the other. The resonant peaks in the pumping current should be experimentally observable. We have developed a density matrix approach that describes the dynamics of the system on time scales much larger than the period of the applied irradiation. In contrast to previous works we additionally consider the case of slow modulation of the irradiation amplitude. Harmonic modulation produces additional sidepeaks in the photoresponse, and pulsed modulation can be used to resolve the Rabi frequency in the time-averaged current.
\end{abstract}

DOI: $10.1103 /$ PhysRevB.63.165313

PACS number(s): 85.35.Be, 73.61.-r, 85.30.Mn, 73.40.Gk

\section{INTRODUCTION}

In recent years quantum dots have attracted great attention. A quantum dot can be thought of as an artificial atom with adjustable parameters. It is of more than fundamental interest to study its properties under various circumstances, e.g., by transport experiments. ${ }^{1}$ By considering a doublequantum-dot system, the analogy with real atoms can be stretched to include artificial molecules. The analog of the covalent bond is then formed by an electron that coherently tunnels back and forth between the two dots. By applying electromagnetic radiation with a frequency equal to the level detuning in the double-dot system, an electron can undergo so-called spatial Rabi oscillations even when the tunneling matrix element between the dots is small.

Recently, several time-dependent transport measurements on quantum dot systems have been reported, ${ }^{2,3}$ most of them being of a spectroscopic nature. It has also been suggested to construct devices from quantum dots. Examples of such applications are pumps that transfer electrons one by one at zero bias voltage by using time-dependent voltages to raise and lower tunnel barrier heights, ${ }^{4}$ or systems in which coupled quantum dots (or quantum wells) are used for quantum-scale information processing. ${ }^{5}$ Several theoretical models for time-dependent transport ${ }^{6}$ through a double quantum dot have already been proposed. For instance, in Ref. 6 dc transport was considered for arbitrary bias voltage when the signal couples to the gate voltages of the dots. At zero bias voltage the system operates as an electron pump. In Ref. 7 the dc current, controlled by external irradiation, was considered for finite bias voltage. These results were recently generalized to include time-dependent gate and bias voltages and tunnel barriers. ${ }^{8,9}$ In all these works a tunnelingHamiltonian approach was used to incorporate the effects of
Coulomb interaction between electrons on the same and on different dots. It is assumed that the barriers separating the leads and the dots are high and therefore the wave functions have only a small overlap. A different approach would be to use scattering states of electrons that extend through the leads and dots which is appropriate for almost transparent barriers (see, e.g., Ref. 10). However, the effects of Coulomb interaction are not easily taken into account in this approach. Because the transport mechanism in the above mentioned double-dot pumps is determined by sequential tunneling, electrons are pumped incoherently, i.e., the tunneling of an electron into and out of the device are independent events. Also, in these works the time-dependent signal is taken to be a monochromatic.

In this paper we describe, firstly, an new mode of operation of such an electron pump. In this case, electrons can be transferred coherently through the system by means of a cotunneling process. Our device has to be designed in such a way that interdot Coulomb repulsion is important. By appropriately adjusting the gate voltages the device can be made to operate in the co-tunneling regime or the sequential tunneling regime. The latter regime is considered here for comparison with previous works and should be distinguished from the coherent one. In the co-tunneling regime the interdot attraction between an extra electron excited by the ac field into one dot and the hole it left in the other dot stabilizes the excited state. Electrons are prevented from entering or leaving the device independently and inelastic co-tunneling is the lowest nonvanishing order process for transport. This involves correlated tunneling events through the different junctions connecting the dots weakly to the leads. The system switches coherently from the excited state to the ground state each time an electron is pumped through the dots. Alternatively, this process can be seen as coherent transport through 


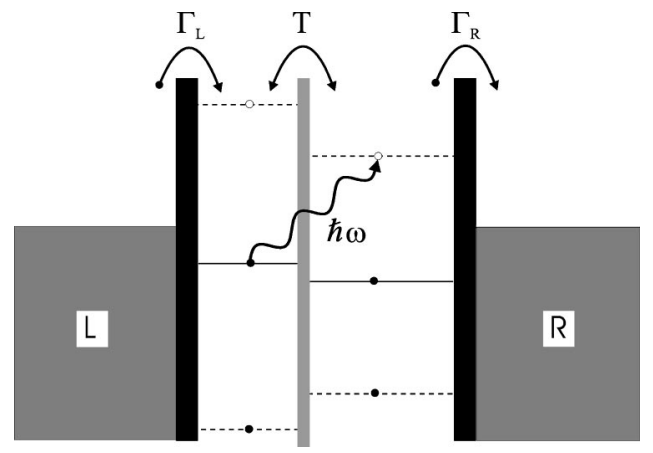

FIG. 1. Double-dot electron pump. The asymmetric gate voltages allow one to induce a photocurrent at zero bias. One can excite an electron from the highest level filled in the left dot to the first empty level of the right dot with an ac field that is resonant with this transition, but not with the corresponding transition in the opposite direction.

a double-quantum-dot qubit. The second main result of this paper is that we predict the effect of slow modulation of the irradiation amplitude on the pumping current. The time scale of the amplitude modulation is assumed to be much larger than the one set by the frequency of the unmodulated signal.

The paper is organized as follows: in Sec. II we introduce the system. In Sec. III we develop the density matrix approach to describe the system only on a time scale much larger than the period of the applied irradiation. This development is similar to that in quantum optics for resonance fluorescence, e.g., Ref. 11, and is central to our treatment of the problems. We apply this approach to the transport through the double dot in the sequential and the co-tunneling regime. In Sec. IV we consider the case where the amplitude of the applied radiation is modulated on the large time scale. Finally, a summary and conclusions are presented in Sec. V.

\section{DOUBLE-DOT ELECTRON PUMP}

The system we consider consists of two coherently coupled quantum dots 1 and 2 connected by tunnel barriers to large reservoirs $L$ and $R$, as depicted in Fig. 1. The leads are assumed to have a continuous electronic energy spectrum. The fixed difference between the electrochemical potentials of the leads $\mu_{L}=\mu-e V_{L_{1}}$ and $\mu_{R}=\mu-e V_{L_{2}}$ (e $>0)$ and the temperature are the smallest two energy scales of the problem. We can thus take them to be zero. Each quantum dot $i=1,2$, contains some number $N_{i}$ electrons and is assumed to be in the ground state. We will concentrate on transitions between the ground states of the individual dots with different numbers of electrons.

Disregarding all tunneling for the moment, let us consider the energy of the double dot within the standard Coulomb blockade model. The capacitive coupling between dot $i$ $=1,2$ and the attached electrodes is taken into account by the gate capacitance $c_{G_{i}}$ and the lead capacitance $c_{L_{i}}$. The mutual capacitive coupling between the dots is described by the interdot capacitance $c_{12}$. The total energy of the double-dot system, when dot 1 and dot 2 are respectively in the $N_{1}$ and $\mathrm{N}_{2}$ electron ground state, reads (see, e.g., Ref. 9):

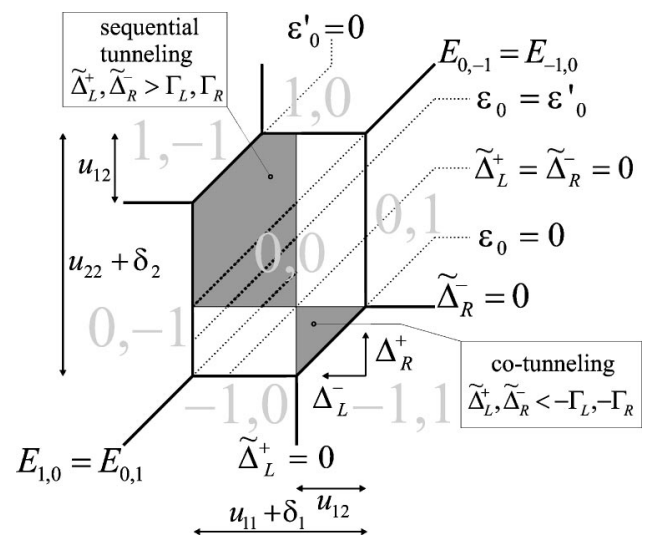

FIG. 2. Stability diagram of the double-dot system near the region where the state $|0,0\rangle$ is stable, with $N_{1}$ and $N_{2}$ electrons in dot 1 and 2, respectively. Along the horizontal and vertical axis the sequential tunneling barrier $\Delta_{L}^{-}$and $\Delta_{R}^{+}$, respectively, are varied independently.

$$
E_{N_{1}, N_{2}}=\sum_{i=1,2} \sum_{l=1}^{N_{i}} \varepsilon_{i l}+\sum_{i=1,2} \frac{1}{2} u_{i i} N_{i}\left(N_{i}-1\right)+u_{12} N_{1} N_{2} .
$$

In the first term, $\varepsilon_{i l}$ is the $l$ th effective single particle energy, $l=1, \ldots, N_{i}$ in $\operatorname{dot} i=1,2$ :

$$
\varepsilon_{i l}\left(V_{G_{1}}, V_{G_{2}}\right)=\varepsilon_{i l}^{0}+\frac{1}{2} u_{i i}-e \sum_{j=1,2}\left(\alpha_{i G_{j}} V_{G_{j}}+\alpha_{i L_{j}} V_{L_{j}}\right) .
$$

This incorporates the bare single particle energy $\varepsilon_{i l}^{0}$, a conveniently chosen offset $u_{i i} / 2$ and the linear shift with the electrode voltages. The coefficients of the voltages $\alpha_{i G_{j}}$ $=C_{i j}^{-1} c_{G_{j}}, \alpha_{i L_{j}}=C_{i j}^{-1} c_{L_{j}}$ depend on the dot-electrode capacitances and the inverse capacitance matrix elements

$$
C_{i i}^{-1}=\frac{\left(c_{1} c_{2}\right) / c_{i}}{c_{1} c_{2}-c_{12}^{2}}, \quad C_{12}^{-1}=C_{21}^{-1}=\frac{c_{12}}{c_{1} c_{2}-c_{12}^{2}},
$$

where $c_{i}=c_{G_{i}}+c_{L_{i}}+c_{12}$ is the total capacitance of dot $i$ $=1,2$. By appropriately changing the gate voltages the effective single particle levels in dot 1 and 2 can be independently shifted with respect to each other. In the second and third term in Eq. (1), $u_{i i}=e^{2} C_{i i}^{-1}$ is the intradot charging energy of dot $i=1,2$ and $u_{12}=e^{2} C_{12}^{-1}<u_{11}, u_{22}$ the interdot charging energy.

Let us now consider the stability of a ground state of the double dot with respect to the tunneling between the individual dots and the leads. Assume that the gate voltages $V_{G_{1}}, V_{G_{2}}$ are such that in the stable state of the double dot there are $N_{1}$ and $N_{2}$ electrons in, respectively, dot 1 and 2 and denote this state by $|0,0\rangle$. The stability diagram of the double dot near the region were this state is stable is sketched in Fig. 2 for the typical case where interdot charging is important: $u_{12} \leqslant u_{11}, u_{22}$. The region of stability for state $|0,0\rangle$ has a hexagonal shape. The stable states $\left|n_{1}, n_{2}\right\rangle$ in the six neighboring regions have $N_{1}+n_{1}$ and $N_{2}+n_{2}$ elec- 
trons in dot 1 and 2, respectively, where $n_{1} \neq n_{2}=0, \pm 1$ and $\left|n_{1}+n_{2}\right| \leqslant 1$. The energies of these states, $E_{n_{1}, n_{2}}, n_{i}=0,1$, are found from the right-hand-side of (1) by replacing $N_{i} \rightarrow N_{i}$ $+n_{i}$. The hexagonal region is bounded by six stability constraints for state $|0,0\rangle$. The first four constraints follow from the requirement that the energy barrier for injection $(+)$ or emission (-) of an electron to or from either lead,

$$
\begin{gathered}
\Delta_{L}^{+}=E_{1,0}-\mu-E_{0,0}=\varepsilon_{1 N_{1}+1}+u_{11} N_{1}+u_{12} N_{2}-\mu, \\
\Delta_{R}^{+}=E_{0,1}-\mu-E_{0,0}=\varepsilon_{2 N_{2}+1}+u_{22} N_{2}+u_{12} N_{1}-\mu, \\
\Delta_{R}^{-}=E_{0,-1}+\mu-E_{0,0}=\mu-\left(\varepsilon_{2 N_{2}}+u_{22}\left(N_{2}-1\right)+u_{12} N_{1}\right), \\
\Delta_{L}^{-}=E_{-1,0}+\mu-E_{0,0}=\mu-\left(\varepsilon_{1 N_{1}}+u_{11}\left(N_{1}-1\right)+u_{12} N_{2}\right),
\end{gathered}
$$

should be positive. Sufficiently far away from the four lines $\Delta_{L, R}^{ \pm}=0$ i.e. $\Delta_{L, R}^{ \pm} \gg \Gamma_{L, R}$ the sequential tunneling of single electrons through the junctions connecting a dot and lead is suppressed; cf. Fig. 2. Here the typical tunnel rate is $\Gamma_{L, R}$ $=2 \pi v_{L, R}\left|t_{L, R}\right|^{2}$, where $v_{L, R}$ is the density of states in the left and right lead, respectively, and $t_{L, R}$ is the matrix element between the states in the lead and in the dot, which depends only weakly on the energy. Two of the sequential tunneling barriers (2) can be independently tuned by the gate voltages, the other two are related to these by $\Delta_{L}^{+}+\Delta_{L}^{-}$ $=u_{11}+\delta_{1}$ and $\Delta_{R}^{+}+\Delta_{R}^{-}=u_{22}+\delta_{2}$ where $\delta_{i}=\varepsilon_{i N_{i}+1}-\varepsilon_{i N_{i}}$ $=\varepsilon_{i N_{i}+1}^{0}-\varepsilon_{i N_{i}}^{0}$ is the spacing between the two "active" single particle levels in dot $i=1,2$. It will be convenient from here on to consider $0<\Delta_{L}^{-}<u_{11}+\delta_{1}$ and $0<\Delta_{R}^{+}<u_{22}+\delta_{2}$ as independent variables instead of the two gate voltages; cf. Fig. 2. Two additional stability constraints follow from the requirement that the energy barriers for polarizing the double dot with respect to state $|0,0\rangle$,

$$
\begin{aligned}
& \varepsilon_{0}=E_{-1,1}-E_{0,0}=\Delta_{L}^{-}+\Delta_{R}^{+}-u_{12}, \\
& \varepsilon_{0}^{\prime}=E_{1,-1}-E_{0,0}=\Delta_{L}^{+}+\Delta_{R}^{-}-u_{12},
\end{aligned}
$$

should be positive. Here $\Delta_{L}^{\mp}, \Delta_{R}^{ \pm}$are the positive Coulomb energies we must pay to change the number of electrons on each dot from the stable configuration $|0,0\rangle$, and $-u_{12}$ is the energy we gain by creating an attracting electron-hole pair with respect to the stable state $|0,0\rangle$. Note that the former energies also depend on $u_{12}$, i.e., they incorporate the interaction between the extra electron or hole and the electrons in both dots. Sufficiently far from the lines $\varepsilon_{0}=0$ and $\varepsilon_{0}^{\prime}=0$ (cf. Fig. 2), the polarization of the double dot by a coherent co-tunneling process is suppressed: $\varepsilon_{0}, \varepsilon_{0}^{\prime} \gg \Gamma_{\mathrm{ct}}^{\prime}$ where $\Gamma_{\mathrm{ct}}^{\prime}$ $\ll \Gamma_{L, R}$ is some typical rate for this process. In such a secondorder process an electron is injected into one dot and another electron is emitted from the other dot, effectively transporting one charge across the double dot. From the relation $\varepsilon_{0}$ $+\varepsilon_{0}^{\prime}=\Sigma_{i=1,2}\left(u_{i i}-u_{12}+\delta_{i}\right)>0$ we find that $\varepsilon_{0}, \varepsilon_{0}^{\prime}>0$ corresponds to $u_{12}<\Delta_{L}^{-}+\Delta_{R}^{+}<\Sigma_{i=1,2}\left(u_{i i}-u_{12}+\delta_{i}\right)+u_{12}$ in the stability diagram.
Now consider the coherent tunneling of electrons between the dot 1 and 2. If the co-tunneling barriers are larger than the matrix element $T$ for this process, $\varepsilon_{0}, \varepsilon_{0}^{\prime} \gg T$, then the polarization of the double dot by coherent tunneling of an electron between the dots is also suppressed. Under these conditions the dc transport through the double dot is blocked at low bias voltage, i.e., we have the Coulomb blockade.

This situation is changed, however, if we apply electromagnetic radiation to the system. Assume that a timedependent oscillating signal is present on the gate electrodes which will shift the energies of single levels without altering the wave functions too much, so that the time-dependent energy difference between states $|0,0\rangle$ and $|-1,1\rangle$ becomes

$$
\varepsilon(t)=\varepsilon_{0}+V \cos \omega t,
$$

where $V$ is the amplitude and $\omega$ the frequency of the externally applied signal. When the frequency of this applied radiation matches the time-independent energy difference $\varepsilon_{0}$ between states $|0,0\rangle$ and $|-1,1\rangle$, it is possible for an electron from the left dot to tunnel to the right one by absorbing one energy quantum $\omega \approx \varepsilon_{0}$ from the field. In principle, this electron can now leave the system by tunneling to the right lead, resulting in the state $|-1,0\rangle$. An electron from the left lead can then tunnel to the left dot, thus restoring the ground state. Effectively, an electron has now been transferred from the left electrode to the right one. Alternatively, the electron can coherently tunnel back by emitting an energy quantum resulting in state $|0,0\rangle$. This transport cycle, $|0,0\rangle \leftrightarrow|-1,1\rangle$ $\rightarrow|-1,0\rangle \rightarrow|0,0\rangle$, is not the only one. Another possible sequence, in which the system passes the intermediate state $|0,1\rangle$, is given by $|0,0\rangle \leftrightarrow|-1,1\rangle \rightarrow|0,1\rangle \rightarrow|0,0\rangle$. The three other states can be disregarded under the following conditions. First, the field should not be resonant with the transition to the other excited state $|1,-1\rangle$ : $\left|\varepsilon_{0}-\omega\right| \ll\left|\varepsilon_{0}^{\prime}-\omega\right|$. This is the case when the distance between the two excited levels is much larger than the detuning of the frequency, $\left|\varepsilon_{0}^{\prime}-\varepsilon_{0}\right| \gg|\delta \omega|=\left|\varepsilon_{0}-\omega\right|$, which, in the stability diagram, corresponds to [cf. Eqs. (3a), (3b)]

$$
\left|2\left(\Delta_{L}^{-}+\Delta_{R}^{+}\right)-\sum_{i=1,2}\left(u_{i i}+\delta_{i}\right)\right| \gg|\delta \omega| .
$$

Second, the states $|1,0\rangle,|0,-1\rangle$ should not be resonant with intermediate states of the transport cycle $|0,1\rangle$ and $|-1,0\rangle$, respectively, $\left|E_{1,0}-E_{0,1}\right| \gg T$ and $\left|E_{0,-1}-E_{-1,0}\right| \gg T$ corresponding to

$$
\begin{aligned}
& \left|u_{11}+\delta_{1}-\left(\Delta_{L}^{-}+\Delta_{R}^{+}\right)\right| \gg T, \\
& \left|u_{22}+\delta_{2}-\left(\Delta_{L}^{-}+\Delta_{R}^{+}\right)\right| \gg T .
\end{aligned}
$$

Thus under these conditions an electron can be excited from the left dot into the right one, but the probability of exciting an electron from the right dot to the left dot can be disregarded.

The details of the transport mechanism of a pumping cycle depend on the energies of the intermediate states $|-1,0\rangle$ and $|0,1\rangle$ relative to the pumped state $|-1,1\rangle$ as shown in Fig. 3 which are controlled by the gate voltages. 


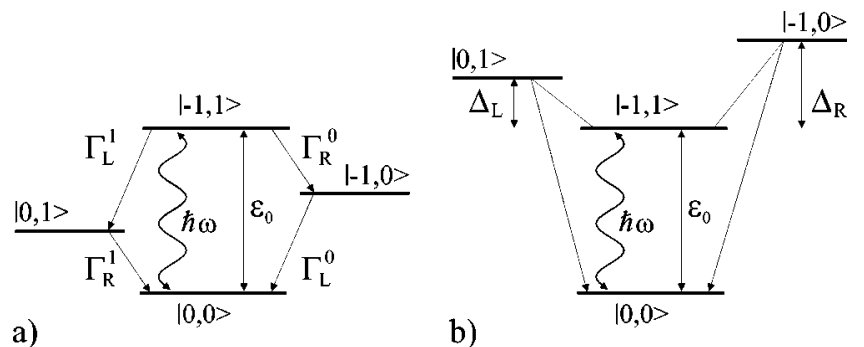

FIG. 3. Energy diagrams of the double dot electron pump together with the leads: (a) sequential tunneling regime, (b) cotunneling regime. The Coulomb interaction between the extra positive charge on the left dot and negative charge on the right dot determines the position of level $E_{-1,1}$ relative to $E_{-1,0}$ and $E_{0,1}$.

The energy barrier for injecting an electron from the left lead into dot 1 and for emitting an electron from the dot 2 dot to the right lead,

$$
\begin{gathered}
\widetilde{\Delta}_{L}^{+}=E_{0,1}-\mu-E_{-1,1}=u_{12}-\Delta_{L}^{-}, \\
\widetilde{\Delta}_{R}^{-}=E_{-1,0}+\mu-E_{-1,1}=u_{12}-\Delta_{R}^{+},
\end{gathered}
$$

can be positive or negative, depending on the position in the stable region of $|0,0\rangle$. Here $u_{12}$ is the energy we must pay to break up the attracting electron-hole pair with respect to state $|0,0\rangle$ and $-\Delta_{L}^{-},-\Delta_{R}^{+}$is the Coulomb energy we gain by changing the number of electrons on one of the dots to the value of the stable configuration $|0,0\rangle$. In this paper we consider two regimes of operation of the double dot electron pump, which are schematically depicted in Fig. 3: (I) both barriers are negative, $\widetilde{\Delta}_{L}^{+}, \widetilde{\Delta}_{R}^{-} \ll-\Gamma_{L},-\Gamma_{R}$ : the pumped level can decay through sequential tunneling processes; (II) both barriers are positive, $\widetilde{\Delta}_{L}^{+}, \widetilde{\Delta}_{R}^{-} \gg \Gamma_{L}, \Gamma_{R}$ : the excited state is stable with respect to sequential tunneling but can decay through an inelastic co-tunneling process. We do not consider the more complicated intermediate case $\left|\widetilde{\Delta}_{L}^{+}\right|,\left|\widetilde{\Delta}_{R}^{-}\right|$ $\lesssim \Gamma_{L}, \Gamma_{R}$ where resonant processes between leads and dots are important. In the stability diagram in Fig. 2 the two regimes correspond to

$$
\begin{gathered}
\text { (I) }\left|\Delta_{L}^{-}-\Delta_{R}^{+}\right|<\left(\Delta_{L}^{-}+\Delta_{R}^{+}\right)-2 u_{12}, \\
2 u_{12}<\Delta_{L}^{-}+\Delta_{R}^{+}<\sum_{i=1,2}\left(u_{i i}-u_{12}+\delta_{i}\right), \\
\text { (II) }\left|\Delta_{L}^{-}-\Delta_{R}^{+}\right|<2 u_{12}-\left(\Delta_{L}^{-}+\Delta_{R}^{+}\right), \\
u_{12}<\Delta_{L}^{-}+\Delta_{R}^{+}<2 u_{12},
\end{gathered}
$$

where $<$ stands for "separated by energy large compared with $\Gamma_{L, R}$.' (6) should be excluded from these regions.

In regime (I) $\left(\widetilde{\Delta}_{L}^{+}, \widetilde{\Delta}_{R}^{-}<0\right)$ the charging of the individual dots dominates the transport. The system relaxes to the ground state via the two sequential (and thus incoherent) tunneling processes described above. As shown in Fig. 3(a), the four tunneling processes are described by the rates
$\Gamma_{L}^{1}, \Gamma_{R}^{1}, \Gamma_{L}^{0}$, and $\Gamma_{R}^{0}$, respectively. The following rate equations describe the density matrix in the sequential tunneling regime:

$$
\begin{gathered}
\partial_{t} \rho_{-10,-10}=+\Gamma_{R}^{0} \rho_{-11,-11}-\Gamma_{L}^{0} \rho_{-10,-10} \\
\partial_{t} \rho_{00,00}=-i T\left(\rho_{-11,00}-\rho_{00,-11}\right)+\Gamma_{L}^{0} \rho_{-10,-10}+\Gamma_{R}^{1} \rho_{01,01}
\end{gathered}
$$

$$
\begin{gathered}
\partial_{t} \rho_{-11,-11}=+i T\left(\rho_{-11,00}-\rho_{00,-11}\right)-\left(\Gamma_{R}^{0}+\Gamma_{L}^{1}\right) \rho_{-11,-11} \\
\partial_{t} \rho_{01,01}=+\Gamma_{L}^{1} \rho_{-11,-11}-\Gamma_{R}^{1} \rho_{01,01} \\
\partial_{t} \rho_{-11,00}=-i T\left(\rho_{00,00}-\rho_{-11,-11}\right)-i \varepsilon(t) \rho_{-11,00} \\
-\frac{1}{2}\left(\Gamma_{L}^{1}+\Gamma_{R}^{0}\right) \rho_{-11,00} .
\end{gathered}
$$

Here, and throughout this paper, units are used such that $\hbar$ $=1$. The diagonal elements give the probabilities for an electron to be in the corresponding states and probability is conserved, i.e., at any time $t$

$$
\rho_{-10,-10}+\rho_{00,00}+\rho_{-11,-11}+\rho_{01,01}=1
$$

The nondiagonal elements $\rho_{-11,00}=\rho_{00,-11}^{*}$ describe the coherence between states $|-1,1\rangle$ and $|0,0\rangle$. In general the tunnel rates $\Gamma_{L}^{0,1}$ and $\Gamma_{R}^{0,1}$ depend on the energy difference between the states of the transition. We can take $\Gamma_{L, R}^{0,1}=\Gamma_{L, R}$ $=2 \pi v_{L, R}\left|t_{L, R}\right|^{2}$ when the density of states $v_{L, R}$ in the left and right lead, respectively, and the matrix element $t_{L, R}$ between the states in the lead and in the dots depend only weakly on the energy. The average current through the system is given by

$$
I / e=\Gamma_{R}^{0} \rho_{-11,-11}+\Gamma_{R}^{1} \rho_{01,01} .
$$

In regime (II) $\left(\widetilde{\Delta}_{L}^{+}, \widetilde{\Delta}_{R}^{-}>0\right)$ the interdot attraction of the electron-hole pair is dominant over the (de)charging of the individual dots. The decay of the excited state $|-1,1\rangle$ via sequential tunneling is blocked as shown in Fig. 3(b). However, transport is still possible via inelastic cotunneling of electrons. ${ }^{12,13}$ When the system is in state $|-1,1\rangle$, two electrons can tunnel simultaneously through different barriers, one going from the left lead to the left dot, and one from the right dot to the right lead. Because in this transport process a state is virtually occupied these two tunneling events cannot be treated independently. The necessary energy is provided by relaxing the system to the ground state $|0,0\rangle$, thereby releasing an energy $E \approx \varepsilon_{0}$. The co-tunneling rate can be calculated with Fermi's golden rule. The relevant matrix element is a sum of matrix elements for the two possible coherent processes which transfer one electron from $L$ to $R$. The co-tunnel rate is obtained by integrating the partial rates for transitions over the different final states of the leads which are assumed to be uncorrelated: 


$$
\begin{aligned}
\Gamma_{\mathrm{ct}} & =2 \pi \nu_{L} \nu_{R} \int_{-\infty}^{\infty} d \varepsilon_{L} \int_{-\infty}^{\infty} d \varepsilon_{R} f\left(\varepsilon_{L}\right)\left[1-f\left(\varepsilon_{R}\right)\right] \\
& \times\left|\frac{t_{L} t_{R}}{\widetilde{\Delta}_{L}^{+}-\varepsilon_{L}}+\frac{t_{L} t_{R}}{\widetilde{\Delta}_{R}^{-}+\varepsilon_{R}}\right|^{2} \delta\left(\varepsilon_{0}+\varepsilon_{L}-\varepsilon_{R}\right) .
\end{aligned}
$$

Here the matrix elements $t_{L, R}$ for tunneling through the left and right barrier and the densities of states $\nu_{L, R}$ in the left and right electrode are assumed to be energy independent. The zero-temperature co-tunnel rate in our electron pump is

$$
\begin{aligned}
\Gamma_{\mathrm{ct}}= & \frac{\Gamma_{L} \Gamma_{R}}{2 \pi}\left[\frac{\varepsilon_{0}}{\left(\widetilde{\Delta}_{L}^{+}+\varepsilon_{0}\right) \tilde{\Delta}_{L}^{+}}+\frac{\varepsilon_{0}}{\widetilde{\Delta}_{R}^{-}\left(\tilde{\Delta}_{R}^{-}+\varepsilon_{0}\right)}\right. \\
& \left.+2 \frac{\ln \left(1+\varepsilon_{0} / \widetilde{\Delta}_{L}^{+}\right)+\ln \left(1+\varepsilon_{0} / \widetilde{\Delta}_{R}^{-}\right)}{\widetilde{\Delta}_{L}^{+}+\tilde{\Delta}_{R}^{-}+\varepsilon_{0}}\right] \\
= & \frac{\Gamma_{L} \Gamma_{R}}{2 \pi} \varepsilon_{0}\left(\frac{1}{\widetilde{\Delta}_{L}^{+}}+\frac{1}{\widetilde{\Delta}_{R}^{-}}\right)^{2}+O\left(\varepsilon_{0}^{2}\right) .
\end{aligned}
$$

Note that from $\varepsilon_{0}=u_{12}-\left(\widetilde{\Delta}_{L}^{+}+\widetilde{\Delta}_{R}^{-}\right)$[cf. Eqs. (3a) and (7)] we observe that within regime (II) we can have $\varepsilon_{0}$ $\sim \widetilde{\Delta}_{L}^{+}, \widetilde{\Delta}_{R}^{-}$. The energy denominators in Eq. (12a) reflect the fact that the tunneling occurs via the virtual occupation of two states. In contrast to the incoherent sequential tunneling mechanism, the only relevant density matrix elements are those between states $|0,0\rangle$ and $|-1,1\rangle$ since states $|-1,0\rangle$ and $|0,1\rangle$ are occupied only virtually. Taking the cotunneling processes into account we obtain the equations of motion for the density matrix elements:

$$
\begin{aligned}
& \partial_{t} \rho_{00,00}=-i T\left(\rho_{-11,00}-\rho_{00,-11}\right)+\Gamma_{\mathrm{ct}} \rho_{-11,-11}, \\
& \partial_{t} \rho_{-11,-11}=+i T\left(\rho_{-11,00}-\rho_{00,-11}\right)-\Gamma_{\mathrm{ct}} \rho_{-11,-11}, \\
& \partial_{t} \rho_{-11,00}=-i T\left(\rho_{00,00}-\rho_{-11,-11}\right)-i \varepsilon(t) \rho_{-11,00} \\
& -\frac{1}{2} \Gamma_{\mathrm{ct}} \rho_{00,-11},
\end{aligned}
$$

where $\rho_{-11,00}=\rho_{00,-11}^{*}$ and the probability is conserved:

$$
\rho_{00,00}+\rho_{-11,-11}=1
$$

The current is

$$
I(t) / e=\Gamma_{\mathrm{ct}} \rho_{-11,-11}(t)
$$

In both regimes the irradiation relaxes the constraint of energy conservation during tunneling by allowing an electron to absorb or emit a multiple of the energy quantum $\omega$. This gives rise to an enhancement of the zero-bias dc component of the current and additionally it introduces fast oscillations with small amplitude which do not interest us. In the next section we show how to extract the slowly varying component of the density matrix from the exact equations (9) and (13), respectively. We point out that above we have writ- ten expressions for the particle current only. The displacement current can be disregarded here since it does not contribute to the dc current.

\section{TIME SCALE SEPARATION}

In this section we consider the dynamics of the two coherently coupled levels $|a\rangle=|0,0\rangle,|b\rangle=|-1,1\rangle$ on time scales much larger than the period of the applied irradiation $t_{\omega}=2 \pi / \omega$. The details of the other states in each regime are only important for the incoherent processes that depend weakly on the time-dependent energy difference between basis states $|a\rangle$ and $|b\rangle$ :

$$
\varepsilon(t)=\varepsilon_{0}+V(t) \cos (\omega t) .
$$

Here we also allow the amplitude $V(t)$ to be modulated on a timescale which is large relative to $t_{\omega}$. The time scale separation for both regimes can be done in the same way. The coherent part of the dynamics of the state of the system depends only on the time-dependent $H(t)=H_{0}(t)+H_{T}$ where

$$
\mathcal{H}_{0}(t)=\frac{1}{2} \varepsilon(t)(|a\rangle\langle a|-| b\rangle\langle b|)
$$

introduces the energy difference between double-dot states with zero extra electrons and $H_{T}$ describes the tunnel coupling between the dots:

$$
\mathcal{H}_{T}=T(|a\rangle\langle b|+| b\rangle\langle a|) .
$$

We assume that the tunneling amplitude is much smaller than the time-independent energy difference $T \ll \varepsilon_{0}$, whereas $V(t)$ can be of arbitrary magnitude. The tunneling to and from the reservoirs brings the system into a mixed state which can only be described by a density operator $\hat{\rho}$ which obeys the Neumann-Liouville equation with dissipative terms added to the right-hand side: ${ }^{14,15}$

$$
\partial_{t} \hat{\rho}=-i[\mathcal{H}, \hat{\rho}]+\mathcal{L}_{\text {inc }} \hat{\rho} .
$$

Since the incoherent part in Eqs. (9) and (13) is invariant under a phase transformation of the nondiagonal elements, we can derive from Eq. (18) a set of equations that describes the dynamics on large timescales by first performing a standard time-dependent basis transformation ${ }^{7}$ on the density matrix. A rapidly varying time-dependent phase factor is absorbed in the nondiagonal elements of $\hat{\rho}$,

$$
\rho_{a b}=\rho_{a b}^{\prime} e^{-i \phi(t)}
$$

and the diagonal elements are left unchanged. In this new basis the generalized Liouville equation for $\hat{\rho}$ takes the same form as Eq. (18) with the same incoherent part and a new Hamiltonian $\mathcal{H}^{\prime}(t)=\mathcal{H}_{0}^{\prime}+\mathcal{H}_{T}^{\prime}(t)$ with a renormalized energy difference and a time-dependent tunnel amplitude

$$
\begin{gathered}
\mathcal{H}_{0}^{\prime}=\frac{1}{2}\left(\varepsilon(t)-\partial_{t} \phi(t)\right)\left(\left|a^{\prime}\right\rangle\left\langle a^{\prime}|-| b^{\prime}\right\rangle\left\langle b^{\prime}\right|\right), \\
\mathcal{H}_{T}^{\prime}(t)=T e^{-i \phi(t)}\left(\left|a^{\prime}\right\rangle\left\langle b^{\prime}|+| b^{\prime}\right\rangle\left\langle a^{\prime}\right|\right) .
\end{gathered}
$$

We choose the phase to be $\phi(t)=n \omega t+(V(t) / \omega) \sin (\omega t)$ to obtain time-independent diagonal elements 


$$
\varepsilon(t)-\partial_{t} \phi(t)=\varepsilon_{0}-n \omega,
$$

which vanish at the $n$-photon resonance $n \omega=\varepsilon_{0}$ in which we are interested. Furthermore, on small time scales the new tunnel matrix element is a periodic function of time and can be expanded in a Fourier series:

$$
\begin{gathered}
\mathcal{H}_{T}^{\prime}(t) \approx \sum_{m=-\infty}^{+\infty} e^{-i(m+n) \omega t} \mathcal{H}_{T_{m}}^{\prime}(t), \\
\mathcal{H}_{T_{m}}^{\prime}(t)=J_{m}\left(\frac{V(t)}{\omega}\right) T\left(\left|a^{\prime}\right\rangle\left\langle b^{\prime}|+| b^{\prime}\right\rangle\left\langle a^{\prime}\right|\right) .
\end{gathered}
$$

Likewise we expand the density operator into rapidly oscillating contributions with amplitudes that vary on large time scales:

$$
\hat{\rho}^{\prime}(t)=\sum_{r=0}^{\infty} \hat{\rho}^{\prime(r)}(t) e^{i r \omega t}
$$

Inserting this into the generalized Liouville equation for $\hat{\rho}^{\prime}$ we obtain an infinite set of coupled equations for the slowly varying coefficients $\hat{\rho}^{\prime(r)}$. The amplitude of the fast oscillations $\hat{\rho}^{(1)}$ is of order $T / \omega \approx T / \varepsilon_{0} \ll 1$ and can be disregarded: the dc component $\rho^{(0)}(t)$ satisfies

$$
\partial_{t} \hat{\boldsymbol{\rho}}^{\prime(0)}=-i\left[\mathcal{H}_{0}^{\prime}+\mathcal{H}_{T_{-n}}^{\prime}, \hat{\boldsymbol{\rho}}^{\prime(0)}\right]+\mathcal{L}_{\text {inc }} \hat{\boldsymbol{\rho}}^{\prime(0)} .
$$

Thus the nearly isolated states $|a\rangle,|b\rangle$ irradiated at a resonant frequency $\omega \approx \varepsilon_{0} / n$ are equivalent to almost degenerate states $\left|a^{\prime}\right\rangle,\left|b^{\prime}\right\rangle$ coupled by a tunneling matrix element

$$
\bar{T}(t)=J_{-n}\left(\frac{V(t)}{\omega}\right) T=(-1)^{n} J_{n}\left(\frac{V(t)}{\omega}\right) T,
$$

which only varies on large time scales. In the following we will only consider the one-photon resonance, i.e., $\omega \approx \varepsilon_{0}$ and we omit the superscripts used above to distinguish the slowly varying components from $\hat{\rho}$ itself. However, the equations can be generalized to the $n$-photon case by replacing $J_{1}(V / \omega) \rightarrow J_{n}(V / \omega)$ and $\varepsilon_{0}-\omega \rightarrow \varepsilon_{0}-n \omega$. Due to the oscillatory behavior of the Bessel function $J_{1}$ the coherent tunneling amplitude can be tuned between $0 \leqslant \bar{T} \leqq 0.58 T$ by varying the amplitude/frequency ratio of the irradiation over a range $0 \leqslant V \leqslant 1.84 \omega$. The vanishing of the effective tunnel matrix element, $\bar{T}=0$ for nonzero $V / \omega$ is one of the features which distinguishes photon-assisted tunneling from adiabatic electron transfer. A similar renormalization of the tunnel coupling to zero is also known from driven double-well potentials. ${ }^{16}$

The approach developed above allows us the extract the slowly varying components of the current in both regimes of operation of the electron pump. We point out that we have only changed the coherent part of the dynamics which is the same in both regimes.

\section{A. Sequential tunneling regime}

Equations (9) describe the dynamics of the double dot in the sequential tunneling regime on the long time scale when we replace $\varepsilon(t) \rightarrow \varepsilon_{0}-\omega, T \rightarrow \bar{T}=-J_{1}(V(t) / \omega) T$. The solution of these equations tends to a stationary value, which is independent of the initial conditions, on typical time scale $\max \left\{\left(\Gamma_{L}^{0,1}\right)^{-1},\left(\Gamma_{R}^{0,1}\right)^{-1}\right\}$. The solution of $\partial_{t} \hat{\rho}(t)=0$ gives a Lorentzian line shape of the current peak as a function of $\varepsilon_{0}-\omega$,

$$
I / e=I_{\max } w^{2} /\left[w^{2}+\left(\varepsilon_{0}-\omega\right)^{2}\right],
$$

where the maximum current $I_{\max }$ and half width at half maximum $w$ are given by

$$
\begin{gathered}
I_{\max } / e=1 /\left\{\frac{1}{\Gamma_{L}^{0}+\Gamma_{R}^{1}}\left[2+\left(\frac{\Gamma_{R}^{0}}{\Gamma_{L}^{0}}+\frac{\Gamma_{L}^{1}}{\Gamma_{R}^{1}}\right)\right]+\frac{\Gamma_{L}^{0}+\Gamma_{R}^{1}}{4 \bar{T}^{2}}\right\}, \\
w=\sqrt{\left[2+\left(\frac{\Gamma_{R}^{0}}{\Gamma_{L}^{0}}+\frac{\Gamma_{L}^{1}}{\Gamma_{R}^{1}}\right)\right] \bar{T}^{2}+\frac{\left(\Gamma_{L}^{0}+\Gamma_{R}^{1}\right)^{2}}{4}} .
\end{gathered}
$$

Let us assume that the tunnel rates for each barrier are the same: $\Gamma_{L, R}^{0,1}=\Gamma_{L, R}$. If the double dot is weakly coupled to the leads i.e. $\Gamma_{L, R} \ll T \ll \varepsilon_{0} \approx \omega$ then one can access the regime $\Gamma_{L, R},\left|\varepsilon_{0}-\omega\right| \ll \bar{T}$ by adjusting the irradiation amplitude to $V \approx 1.84 \omega$, where the coherent state in the double dot dominates the transport properties. The two delocalized states in the double dot are independent channels for transport and the current increases with $\Gamma_{L, R}$ :

$$
I_{\max } / e=1 /\left(\frac{1}{\Gamma_{L}}+\frac{1}{\Gamma_{R}}\right), \quad w=2 \bar{T} .
$$

In the opposite regime $\left|\varepsilon_{0}-\omega\right| \ll \bar{T} \ll \Gamma_{L, R}$, which can be accessed by tuning $V \ll 1.84 \omega$, the decoherence due to tunneling to and from the reservoir dominates the transport. In this case the height of the current peak is proportional to $\bar{T}^{2}$ and decreases with enhanced tunneling $\Gamma_{L, R}$ :

$$
I_{\max } / e=4 \bar{T}^{2} /\left(\Gamma_{L}+\Gamma_{R}\right), \quad w=\left(\Gamma_{L}+\Gamma_{R}\right) / 2 .
$$

The current peak $I_{\max } / e$ reaches a maximum $\bar{T} / 2$ as a function of $\Gamma_{L, R}$ when $\Gamma_{L}=\Gamma_{R}=2 \bar{T}$. This can be understood as the precise matching of tunneling times: the time for half a Rabi oscillation in the double dot is exactly equal to the time for filling the left dot and for emptying the right dot. In Fig. 4(a) we have plotted the maximum current and the width as a function of the tunnel rate $\Gamma$ relative to the tunnel coupling $T$. If the double dot is strongly coupled to the leads i.e. $T$ $\ll \Gamma_{L, R} \ll \varepsilon_{0} \approx \omega$ only the regime $\bar{T},\left|\varepsilon_{0}-\omega\right| \ll \Gamma_{L, R}$ is accessible.

We point out that in the sequential tunneling regime the transport on "large" time scales is equivalent to transport of "free" electrons (i.e., negligible interdot repulsion) through a double dot with renormalized static parameters $\varepsilon_{0} \rightarrow \varepsilon_{0}$ $-\omega, T \rightarrow J_{1}(V / \omega) T, \Gamma_{L, R} \cdot{ }^{15}$ Also, the results here are simi- 


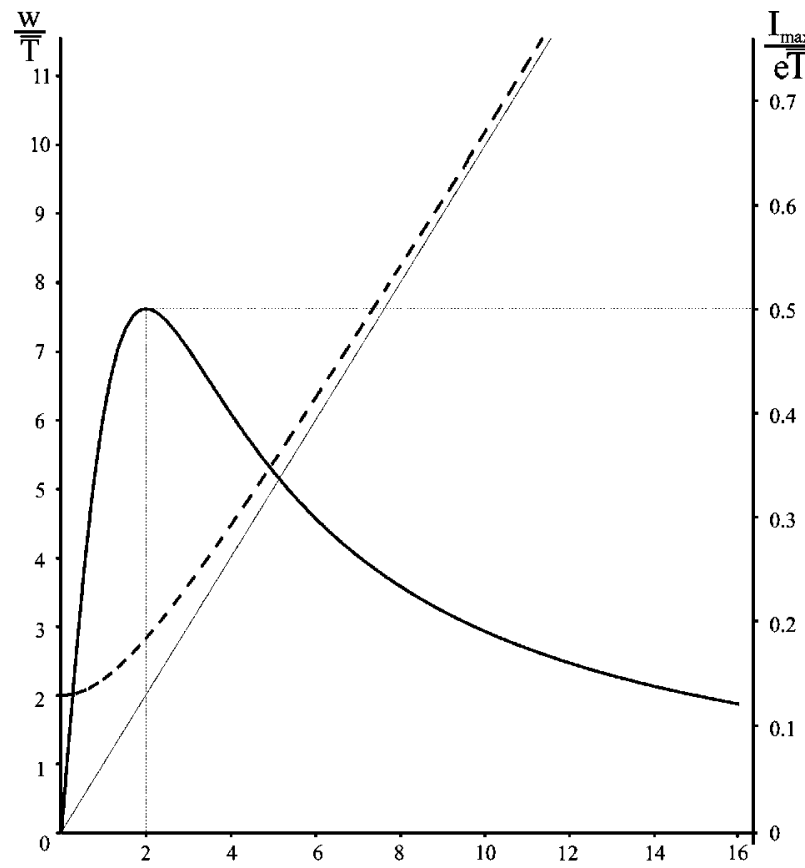

a)

$\frac{\Gamma}{\bar{T}}$

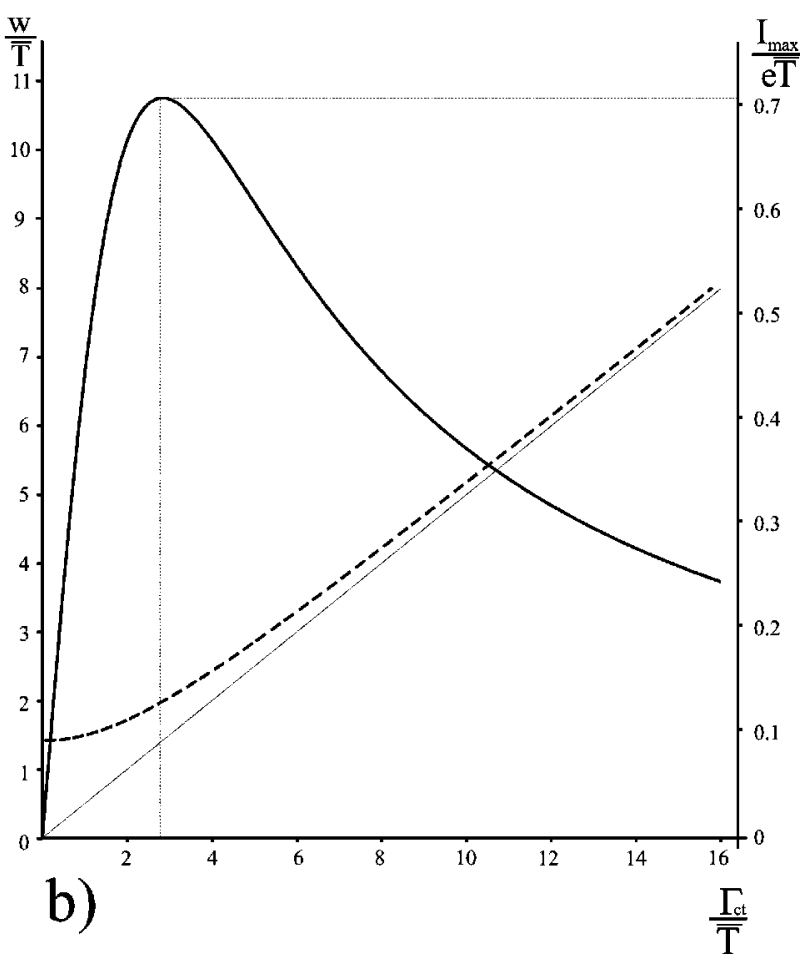

FIG. 4. Lorentzian current peak height (bold solid line) and width (dashed line) as a function of (a) $\Gamma / \bar{T}$ ( $\Gamma=\Gamma_{L}=\Gamma_{R}$ ) in the sequential tunneling regime and of (b) $\Gamma_{\mathrm{ct}} / \bar{T}$ in the cotunneling regime. For fixed $\bar{T}$ the maximal co-tunneling current as a function of $\Gamma_{\mathrm{ct}}$ is larger than the maximal sequential tunneling current as a function of $\Gamma$.

lar to the analytical results obtained for the noninteracting case in Ref. 6, where only sequential tunneling occurs.

\section{B. Co-tunneling regime}

In the co-tunneling regime two well-separated time scales are involved, namely the "long" time $t_{\mathrm{ct}}=\Gamma_{\mathrm{ct}}^{-1}$ between two co-tunneling processes and the "short" time of the process itself $t_{\text {virt }}=\varepsilon_{0}^{-1}$ during which the energy is uncertain by an amount $\varepsilon_{0}$. Near resonance the applied frequency matches the detuning of the levels so $t_{\text {virt }} \approx 2 \pi / \omega=t_{\omega}$. The density matrix approach developed above describes the system on the "large" time scale $t_{\mathrm{ct}}$. Equations (13) describe the dynamics of the double dot in the sequential tunneling regime on the long time scale when we replace $\varepsilon(t) \rightarrow \varepsilon_{0}-\omega, T$ $\rightarrow \bar{T}=-J_{1}(V(t) / \omega) T$. The stationary solution of these equations gives a Lorentzian current in $\varepsilon_{0}-\omega$ with height $I_{\max }$ and half-width $w$ :

$$
\begin{aligned}
I_{\max } / e & =1 /\left(\frac{2}{\Gamma_{\mathrm{ct}}}+\frac{\Gamma_{\mathrm{ct}}}{4 \bar{T}^{2}}\right), \\
w & =\sqrt{2 \bar{T}^{2}+\Gamma_{\mathrm{ct}}^{2} / 4} .
\end{aligned}
$$

For weak coupling to the reservoirs $\Gamma_{\mathrm{ct}} \ll \bar{T}$ the peak height is constant and the width increases linearly with the coherent coupling $\bar{T}$ :

$$
I_{\max } / e=\frac{1}{2} \Gamma_{\mathrm{ct}}, \quad w=\sqrt{2} \bar{T} .
$$

In the opposite case $\Gamma_{\mathrm{ct}} \gg \bar{T}$ the peak height is small but increases rapidly with $\bar{T}$ whereas now the width is constant:

$$
I_{\max } / e=4 \bar{T}^{2} / \Gamma_{\mathrm{ct}}, \quad w=\frac{1}{2} \Gamma_{\mathrm{ct}} .
$$

The current peak $I_{\max } / e$ reaches a maximum $\bar{T} / \sqrt{2}$ as a function of $\Gamma_{\mathrm{ct}}$ when $\Gamma_{\mathrm{ct}}=2 \sqrt{2} \bar{T}$. In Fig. 4(b) the scaled pumping current is plotted for different values of $\Gamma_{\text {ct }} / \bar{T}$. In the cotunneling regime electrons are transferred through strongly correlated transport channels. Therefore the condition for the maximum current cannot be understood as a the precise matching of tunneling times as in the sequential tunneling regime (factor $\sqrt{2}$ ). Equations (13) coincide with those that describe the transport of electrons that are correlated by strong Coulomb repulsion through a double dot at high voltage bias, with renormalized static parameters $\varepsilon_{0} \rightarrow \varepsilon_{0}-\omega$, $T \rightarrow J_{1}(V / \omega) T, \Gamma_{\mathrm{ct}} \rightarrow \Gamma_{R}$ in the limit where the tunneling into the left dot is so fast, $\Gamma_{L} \gg \Gamma_{R}, T$, that the current no longer depends on it. ${ }^{7}$ In this system the correlation of the conduction channels prevents more than one channel from being occupied and reduces the effective tunnel rate by a factor of 2 in Eq. (33). ${ }^{17}$ This blockade can be obtained formally from the density matrix equations (9) of the "free" electrons by taking $\Gamma_{L, R}^{1} \rightarrow 0$ and $\Gamma_{L}^{0} \gg \Gamma_{R}^{0}=\Gamma_{\mathrm{ct}}$.

Comparing the co-tunneling and sequential tunneling regime the main difference is that for fixed $\Gamma_{L, R}$ the co-tunnel rate $\propto \Gamma_{L} \Gamma_{R}$ is much smaller: $\Gamma_{\mathrm{ct}} \ll \Gamma_{L, R}$. For $\Gamma_{L, R} \sim \bar{T}$ the sequential tunneling current can be near its maximal value $\sim 2 \bar{T}$, whereas the co-tunneling current will be $\sim \Gamma_{\mathrm{ct}} \ll \bar{T}$. 
However, for $\Gamma_{L, R} \gg \bar{T}$ the sequential tunneling current is much smaller than $\bar{T}$ and it is possible to adjust $\Gamma_{\mathrm{ct}} \sim \bar{T}$ so that the co-tunneling current takes its maximal value $\sim 2 \sqrt{2} \bar{T}$ which is much larger. Thus for fixed $\bar{T}$ the maximal co-tunneling current is larger by a factor $\sqrt{2}$ than the maximal sequential tunneling current (Fig. 4). The width of the co-tunnel peak is also smaller than in the sequential tunneling regime.

\section{MODULATED IRRADIATION OF A DOUBLE DOT}

In this section we consider the sequential tunneling regime already discussed in Sec. III A and apply our approach for large time scales to the cases of pulsed and slow sinusoidal modulation of the irradiation amplitude.

\section{A. Response to irradiation pulses}

By means of irradiation pulses quantum states in the dots can be manipulated. Assuming the double dot to be in the ground state

$$
\rho_{00,00}=1, \rho_{-10,-10}=\rho_{01,01}=\rho_{-11,-11}=\rho_{-11,00}=\rho_{00,-11}=0
$$

at $t=0 \quad$ we solve Eqs. (9) with $\varepsilon(t) \rightarrow \varepsilon_{0}-\omega, T \rightarrow \bar{T}$ $=-J_{1}(V(t) / \omega) T$ for the time evolution under the influence of the irradiation for the case $\Gamma_{L, R}^{0,1}=\Gamma$ :

$$
I(t)=I\left[1-e^{-\Gamma t}\left(\cos \left(\Omega_{\mathrm{R}} t\right)+\frac{\Gamma}{\Omega_{\mathrm{R}}} \sin \left(\Omega_{\mathrm{R}} t\right)\right)\right],
$$

where $\Omega_{\mathrm{R}}=\sqrt{\left(\varepsilon_{0}-\omega\right)^{2}+4 \bar{T}^{2}}$ is the Rabi frequency. For $t$ $\gg \Gamma^{-1}$ the solution tends to the stationary current (29) derived before:

$$
I / e=1 /\left(\frac{1}{\Gamma} \frac{\Omega_{\mathrm{R}}^{2}}{2 \bar{T}^{2}}+\frac{\Gamma}{2 \bar{T}^{2}}\right) .
$$

When the irradiation is switched off at $t=\tau_{p}$, the current decays exponentially as $e^{-\Gamma\left(t-\tau_{p}\right)}$ to zero, as can also be seen by solving the equations for the case $\omega=\bar{T}=0$. One can resolve the Rabi oscillations in the dc current by considering the current averaged over a series of identical pulses ${ }^{18}$ with delay $\tau_{d}$, as a function of the pulse length $\tau_{p}$ (see inset of Fig. 5):

$$
I_{\mathrm{dc}}\left(\tau_{p}\right)=\frac{1}{\tau_{d}}\left(\int_{0}^{\tau_{p}} I(t) d t+I\left(\tau_{p}\right) \int_{\tau_{p}}^{\tau_{d}} e^{-\Gamma\left(t-\tau_{p}\right)} d t\right) .
$$

Here $\tau_{d}-\tau_{p} \gg \Gamma^{-1}$ to ensure that the system is prepared in the ground state (35) at the beginning of the pulse. In the physically interesting case of weak coupling to the leads $\Gamma$ $\ll \Omega_{\mathrm{R}}$ we obtain

$$
I_{\mathrm{dc}}\left(\tau_{p}\right) \approx I \frac{1}{\tau_{d}}\left[\tau_{p}+\frac{1-e^{-\Gamma \tau_{p}} \cos \left(\Omega_{\mathrm{R}} \tau_{p}\right)}{\Gamma}\right] .
$$

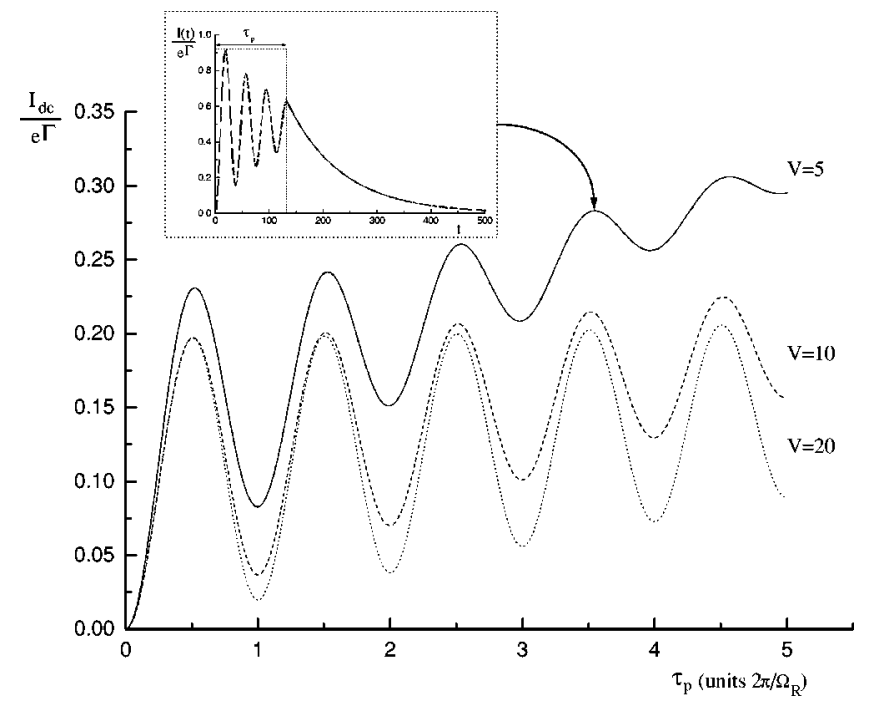

FIG. 5. Double dot subjected to an irradiation pulse train. Current averaged over a series of identical pulses, in units of number of electrons transferred per pulse, as a function of the pulse length for $\varepsilon_{0}=\omega=30, T=1, \Gamma=0.1$ and several irradiation amplitudes $V$. Inset: time-dependent current during one pulse.

The oscillations are most clearly resolvable for $\tau_{p} \ll \Gamma^{-1}$ (Fig. 5). The period of the coherent oscillation at resonance $\omega=\varepsilon_{0}, 2 \pi / \Omega_{\mathrm{R}}=\pi /\left[J_{1}\left(V_{0} / \omega\right) T\right]$, can be tuned by varying the irradiation power $V_{0}$.

\section{B. Sinusoidal amplitude modulation}

Now consider the case where the amplitude of the irradiation is slowly sinusoidally modulated with small modulation amplitude $\widetilde{V} \ll V_{0}$ :

$$
V(t)=V_{0}+\widetilde{V} \cos (\Omega t) .
$$

The case where the modulation amplitude is of the order of or larger than the irradiation amplitude is physically not very interesting because the system then exhibits trivial Fourier peaks at $\omega, \omega+\Omega$ and $\omega-\Omega$ as a function of $\varepsilon_{0}$ in the dc current. To find an analytical solution, we rewrite Eqs. (9) with $\varepsilon(t) \rightarrow \varepsilon_{0}-\omega, T \rightarrow \bar{T}=-J_{1}(V(t) / \omega) T$ in matrix notation:

$$
\frac{\partial \vec{\rho}}{\partial t}=(\hat{\Gamma}+\hat{T}) \vec{\rho}+\vec{c}
$$

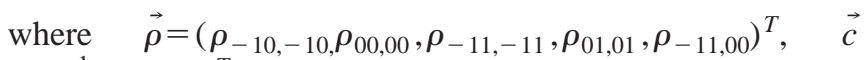
$=\left(\Gamma_{R}^{1}, 0,0,0,0\right)^{T}$ and expand the Bessel function $J_{1}(V(t) / \omega)$ in a Taylor series to second order in $\widetilde{V}$. If we now consider the Fourier coefficients $\vec{\rho}_{n}$ of $\vec{\rho}(t)$ and $T_{n}$ of $J_{1}(V(t) / \omega) T$, we find the following equations for the dc component and first harmonic, if we disregard higher harmonics,

$$
\begin{gathered}
0=\left(\hat{\Gamma}+T_{0} \hat{T}\right) \vec{\rho}_{0}+T_{1} \hat{T}\left(\vec{\rho}_{+1}+\vec{\rho}_{-1}\right)+\vec{c}, \\
\vec{\rho}_{ \pm 1}=-T_{1}\left(\hat{\Gamma}+T_{0} \hat{T} \mp i \Omega \hat{I}\right)^{-1} \hat{T} \vec{\rho}_{0},
\end{gathered}
$$




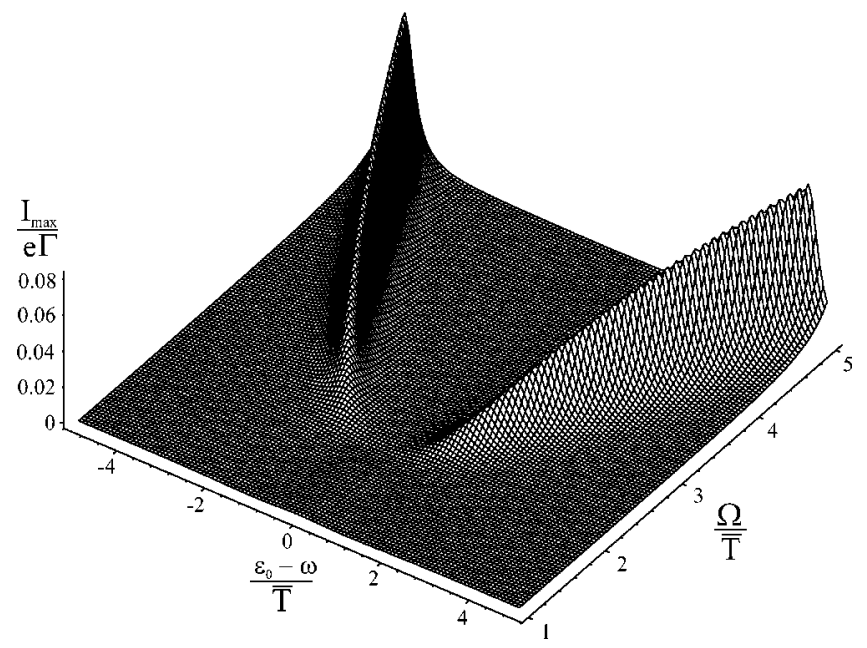

FIG. 6. Electron pump with harmonically amplitude-modulated irradiation. Current as a function of the renormalized level detuning (resonance mismatch of basis frequency) and the frequency of the amplitude modulation for $T=1, \Gamma_{L, R}=0.2, V_{0}=30, \widetilde{V}=10$.

from which we can solve for the dc component $\vec{\rho}_{0}$. If we furthermore assume that $\Gamma_{L, R}^{0,1}=\Gamma \ll \bar{T}$, the solution shows additional side peaks in the photoresponse of the system, which are in good approximation Lorentzians along the hyperbola $\varepsilon_{0}-\omega=\sqrt{\Omega^{2}-4 \bar{T}^{2}}$, i.e., $\Omega=\Omega_{\mathrm{R}}$ as plotted in Fig. 6. The height of these peaks is

$$
\frac{I_{\max }}{e}=\frac{\Gamma}{2} \frac{\left(\alpha^{2}-4\right)^{2} \bar{T}^{\prime} 2 \frac{\widetilde{V}^{2}}{\omega^{2}}}{\alpha^{2}\left(\alpha^{2} \Gamma^{2}+\left(\alpha^{2}-4\right) \bar{T}^{\prime} 2 \frac{\widetilde{V}^{2}}{\omega^{2}}\right)},
$$

with $\alpha=\Omega / \bar{T}, \bar{T}^{\prime}=J_{1}^{\prime}\left(V_{0} / \omega\right) T$, and the half-width at half maximum is

$$
w=\sqrt{\bar{T}^{\prime} \frac{\widetilde{V}^{2}}{\omega^{2}}+\frac{\alpha^{2}}{\alpha^{2}-4} \Gamma^{2}} .
$$

These side peaks thus resolve the Rabi splitting as described in Refs. 6 and 19 in terms of quasi-energies. The height of these peaks can be of the order of the first satellite peak and should therefore be experimentally observable. Notice that at $\varepsilon_{0}=\omega, \Omega=2 \bar{T}$ the width diverges; however, the current peak at this point vanishes since the exact matching $\varepsilon_{0}=\omega$ gives a resonance to which the modulation cannot add extra current.

Thus by applying a high frequency $\omega$ we reduce the energy spacing by a large amount, $\varepsilon_{0} \rightarrow \varepsilon_{0}-\omega$, and modify the tunneling matrix element with an intensity- $(V)$ dependent factor, $T \rightarrow \bar{T}$. The lower frequency $\Omega$ allows one to precisely match the remaining small energy difference $\varepsilon_{0}-\omega$ without significantly altering the tunneling matrix element $\bar{T}$, thereby inducing a photocurrent.

\section{CONCLUSIONS}

We have considered an electron pump consisting of an double quantum dot subject to irradiation. An incoherent and a new coherent pumping mechanism were discussed. We have derived equations of motion for the density matrix elements of the double-dot system that are time-averaged over an interval which is long compared to the period of the applied signal. From these equations we calculated the pumping current in both regimes. In both cases the current peak is a Lorentzian. Surprisingly, for fixed effective tunnel coupling the maximal pumping current in the co-tunneling regime is larger by a factor $\sqrt{2}$ compared to the value in the sequential tunneling regime, where the maximum occurs at a different value of the tunnel rates for each regime. Experimental realization of this device would allow for a systematic study of coherent transport through a solid-state qubit. Moreover, modulation of the irradiation amplitude exhibits interesting phenomena: a train of short pulses should resolve the Rabi frequency in the time-averaged current as a function of the pulse length, and sinusoidal amplitude modulation should provide a tool to resolve the Rabi splitting.

\section{ACKNOWLEDGMENTS}

It is a pleasure to acknowledge useful discussions with Gerrit Bauer, Arne Brataas, Leo Kouwenhoven, and Wilfred van der Wiel. This work was part of the research program of the "Stichting voor Fundamenteel Onderzoek der Materie" (FOM), which was financially supported by the "Nederlandse Organisatie voor Wetenschappelijk Onderzoek" (NWO) and the NEDO project NTDP-98.
${ }^{1}$ L.P. Kouwenhoven and P.L. McEuen, in Nano-Science and Technology, edited by G. Timp (AIP Press, New York, 1996), and references therein.

${ }^{2}$ L.P. Kouwenhoven, S. Jauhar, K. McCormick, D. Dixon, P.L. McEuen, Yu.V. Nazarov, N.C. van der Vaart, and C.T. Foxon, Phys. Rev. B 50, 2019 (1994); L.P. Kouwenhoven, S. Jauhar, J. Orenstein, P.L. McEuen, Y. Nagamune, J. Motohisa, and H. Sakaki, Phys. Rev. Lett. 73, 3443 (1994); Y. Nakamura, C.D. Chen, and J.S. Tsai, ibid. 79, 2328 (1997); T.H. Oosterkamp, L.P. Kouwenhoven, A.E.A. Koolen, N.C. van der Vaart, and C.J.P.M. Harmans, ibid. 78, 1536 (1997).
${ }^{3}$ T.H. Oosterkamp, T. Fujisawa, W.G. van der Wiel, K. Ishibashi, R.V. Hijman, S. Tarucha, and L.P. Kouwenhoven, Nature (London) 395, 873 (1998).

${ }^{4}$ L.P. Kouwenhoven, A.T. Johnson, N.C. van der Vaart, and C.J.P.M. Harmans, and C.T. Foxon, Phys. Rev. Lett. 67, 1626 (1991); F.W.J. Hekking and Yu.V. Nazarov, Phys. Rev. B 44, 9110 (1991).

${ }^{5}$ R. Landauer, Science 272, 1914 (1996); Adriano Barenco, David Deutsch, Artur Ekert, and Richard Jozsa, Phys. Rev. Lett. 74, 4083 (1995); D. Loss and D.P. DiVicenzo, Phys. Rev. A 57, 120 (1998). 
${ }^{6}$ Time-dependent transport in mesoscopic structures is discussed in M. Büttiker, cond-mat/9909126 (unpublished).

${ }^{7}$ C.A. Stafford and N.S. Wingreen, Phys. Rev. Lett. 76, 1916 (1996).

${ }^{8}$ T.H. Stoof and Yu.V. Nazarov, Phys. Rev. B 53, 1050 (1996).

${ }^{9} \mathrm{Ph}$. Brune, C. Bruder, and H. Schoeller, Phys. Rev. B 56, 4730 (1997).

${ }^{10}$ R. Ziegler, C. Bruder, and H. Schoeller, Phys. Rev. B 62, 1961 (2000).

${ }^{11}$ M.H. Pedersen and M. Büttiker, Phys. Rev. B 58, 12993 (1998).

${ }^{12}$ D.F. Walls and G.J. Milburn, Quantum Optics (Springer, Berlin, 1994), Chap. 11.
${ }^{13}$ D.V. Averin and A.A. Odintsov, Phys. Lett. A 140, 251 (1989); D.V. Averin and Yu.V. Nazarov, Phys. Rev. Lett. 65, 2446 (1990).

${ }^{14}$ Yu.V. Nazarov, Physica B 189, 57 (1993).

${ }^{15}$ S.A. Gurvitz and Ya.S. Prager, Phys. Rev. B 53, 15932 (1996).

${ }^{16}$ For a review of driven quantum tunneling see M. Grifoni and P. Hänggi, Phys. Rep. 304, 229 (1998).

${ }^{17}$ M.R. Wegewijs and Yu.V. Nazarov, Phys. Rev. B 60, 14318 (1999).

${ }^{18}$ Y. Nakamura, Yu.A. Pashkin, and J.S. Tsai, Nature (London) 398, 6730 (1999).

${ }^{19}$ M. Holthaus and D. Hone, Phys. Rev. B 47, 6499 (1993). 